\title{
Factores de mayor influencia en el riesgo cardiovascular y su relación con magnesio sérico y su ingesta
}

\section{Factors with greater influence on cardiovascular risk and its relationship with serum magnesium and its intake}

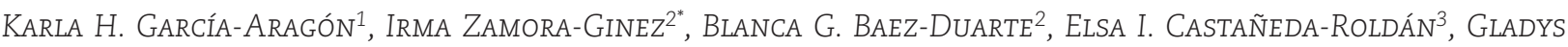 \\ Linares-Fleites ${ }^{1}$, Adriana Nieva-VÁzquez ${ }^{4}$, Paola Meneses-Zamora ${ }^{2}$, Lady K. Pesqueda-Cendejas ${ }^{2}$, NayelLi \\ FLORES-MARTÍNEZ ${ }^{2}$ Y ZAYRA I. BAUTISTA-POT²
}

${ }^{1}$ Facultad de Medicina, Instituto de Ciencias, Ciencias Ambientales; ${ }^{2}$ Facultad de Medicina, Hospital Universitario de Puebla; ${ }^{3}$ nstituto de Ciencias, Ciencias Ambientales, Centro de Investigaciones en Ciencias Microbiológicas; ${ }^{4}$ Complejo Regional Sur. Benemérita Universidad Autónoma de Puebla, Puebla, México

\section{RESUMEN}

En este estudio se caracterizaron 66 sujetos clínica-antropométrica y bioquímicamente. Los factores con mayor influencia identificados fueron: triglicéridos (TG), lipoproteínas de muy baja densidad (VLDL), lipoproteínas de baja densidad (LDL) y colesterol total (CT); Mg sérico (MgS) correlacionó con TG (Rho $=0.270, p=0.028), C T(R h o=0.349, p=0.004)$, LDL (Rho $=0.251, p=0.042)$ y VLDL (Rho $=0.270, p=0.028)$, MgS correlacionó con CT ajustado para índice de masa corporal (IMC), glucosa y edad (Rho $=0.303, p=0.016$ ). Los sujetos sin antecedentes familiares de enfermedad cardiovascular (AF_ECV) presentaron mayor ingesta de $\mathrm{Mg}$ $(p=0.023)$ que aquellos con AF_ECV y los hombres tuvieron mayor ingesta de $\mathrm{Mg}$ que las mujeres $(\mathrm{p}=0.005)$. En conclusión, los factores de mayor influencia en el riesgo CV son TG, LDL, VLDL y CT, los cuales correlacionan positiva y significativamente con MgS, CT aún ajustado para IMC, glucosa y edad; además la ingesta de magnesio es mayor en sujetos sin AF_ECV y en los hombres.

Palabras clave: Factores de riesgo cardiovascular. Magnesio sérico. Ingesta de magnesio.

\begin{abstract}
In this study, 66 clinical-anthropometric and biochemical subjects were characterized. The factors with the greatest influence identified were: triglycerides (TG), very low density lipoproteins (VLDL), low density lipoproteins (LDL) and total cholesterol (TC); Serum Mg (SMg) correlated with TG (Rho $=0.270, p=0.028), T C($ Rho $=0.349, p=0.004), L D L$ $($ Rho $=0.251, p=0.042)$ and VLDL (Rho $=0.270, p=0.028)$, SMg correlates with TC adjusted for body mass index (BMI), glucose and age (Rho $=0.303, p=0.016)$; Subjects without a family history of cardiovascular disease (FH_ECV) had a higher intake of $\mathrm{Mg}(\mathrm{p}=0.023)$ than those with $\mathrm{FH}$ ECCV and men had a higher intake of Mg than women ( $p=0.005)$. In conclusion, the factors with the greatest influence on $\mathrm{CV}$ risk are TG, LDL, VLDL and TC, which correlate positively and significantly with serum magnesium, TC still adjusted for BMI, glucose, and age; in addition, magnesium intake is higher in subjects without FH_ECV and in men.
\end{abstract}

Keywords: Cardiovascular risk factors. Serum magnesium. Magnesium intake.
Correspondencia:

*Irma Zamora-Ginez

E-mail: irma.zamora@correo.buap.mx
Fecha de recepción: 16-10-2020

Fecha de aceptación: 10-03-2021

DOI: 10.24875/RME.20000100
Disponible en internet: 22-02-2022

Rev Mex Endocrinol Metab Nutr. 2022;9:3-12

2462-4144 / @ 2021 Sociedad Mexicana de Nutrición y Endocrinología, AC. Publicado por Permanyer. Este es un artículo open access bajo la licencia CC BY-NC-ND (http://creativecommons.org/licenses/by-nc-nd/4.0/). 


\section{INTRODUCCIÓN}

Actualmente, las enfermedades cardiovasculares (ECV) son la principal causa de muerte a en el mundo' ${ }^{1}$. Aunado a esto, entre los pacientes con enfermedad por el coronavirus 2 del síndrome respiratorio agudo (SARS-CoV-2), existe una alta prevalencia de $\mathrm{ECV}^{2}$. En México, de enero a marzo del 2019, la tasa de mortalidad en conjunto por ECV fue del $49.9 \%{ }^{3}$ y durante los primeros tres meses de la pandemia (de marzo a junio del 2020) ha sido asociada con muerte por COVID-19 (5\%) ${ }^{4}$. Las ECV tienen un origen multifactorial, asociado a factores de riesgo cardiovascular (FRC) como hiperlipidemia, edad y sexo asociados al estilo de vida, como el consumo de tabaco, el sedentarismo, el consumo de alcohol, antecedentes familiares de ECV y el tipo de dieta ${ }^{5-7}$. Por otro lado, presentar diabetes aumenta el riesgo de ECV al doble y se considera de alto riesgo si además existe presencia de otras patologías como la hipertensión ${ }^{5}$. Un marcador de trastornos cardiovasculares es la proteína $C$ reactiva ultrasensible (PCR-us), debido a que participa en la liberación de citocinas a nivel del tejido adiposo, y sus cifras elevadas sugieren estados proinflamatorios $y$, en consecuencia, un FRC ${ }^{8,9}$.

Por otra parte, se ha demostrado que tanto el consumo de alimentos con magnesio $(\mathrm{Mg})$ como el $\mathrm{Mg}$ sérico se relacionan inversamente con un riesgo menor del $15 \%$ de ECV en poblaciones de EE.UU., Suecia, Finlandia, Francia, Alemania, Japón y China (riesgo relativo [RR]: 0.85 , intervalo de confianza (IC) del 95\%: 0.78-0.92; $\mathrm{p}<0.001)^{10}$. También es conocido que el Mg puede actuar como un fármaco natural y reducir el colesterol vinculado a lipoproteínas de baja densidad (c-LDL) y los triglicéridos (TG), así como aumentar el colesterol vinculado a lipoproteínas de alta densidad (c-HDL), debido a que regula a la enzima 3-hidroxi-3-metilglutaril-CoA reductasa (HMG-CoA reductasa) ${ }^{11}$. Aunado a lo anterior, la deficiencia de Mg podría afectar la susceptibilidad cardiovascular y la respuesta al SARS-CoV-2, y su ingesta podría influir en el brote de COVID-19 ${ }^{12}$.

Este trabajo tiene como objetivo determinar mediante análisis de componentes principales (ACP) cuáles son los factores clínico-antropométricos y bioquímicos cuantitativos (edad, índice de masa corporal [IMC], tensión arterial sistólica [TAS], tensión arterial diastólica [TAD], glucosa, TG, colesterol total $[C T], c-L D L$, colesterol vinculado a lipoproteínas de muy baja densidad [c-VLDL], c-HDL y PCR-us) que tienen mayor influencia en el riesgo cardiovascular (RCV) en la población de estudio, y establecer el tipo de relación que tienen estos factores influyentes con el $\mathrm{Mg}$ sérico y su ingesta, así como determinar si existen diferencias en la concentración de $\mathrm{Mg}$ sérico y la ingesta de $\mathrm{Mg}$ entre la presencia o ausencia de estados que han sido reportados asociados a mayor RCV (sexo, presencia de tabaquismo, historia familiar de ECV, obesidad, presencia de diabetes y baja actividad física); los resultados obtenidos podrían servir de base para establecer medidas preventivas de RCV particulares.

\section{MATERIAL Y MÉTODOS}

Se diseñó un estudio transversal en 66 sujetos voluntarios hombres y mujeres no embarazadas, de 23 a 70 años. Los resultados son parte del protocolo aprobado por el Comité de Investigación de la Facultad de Medicina (N. ${ }^{\circ}$ 342) de la Benemérita Universidad Autónoma de Puebla (BUAP). Los participantes, quienes fueron personal académico $y$ administrativo de la BUAP, dieron su consentimiento informado por escrito. Se incluyeron sujetos fumadores, pero se excluyeron a aquellos con dependencia a cigarrillos, además se excluyeron sujetos con consumo de alcohol y/o de suplementos vitamínicos en el último año, y/o con ingesta de esteroides, así como a los sujetos con historia de ECV, enfermedad renal, enfermedad hepática y/o enfermedad inflamatoria.

Se realizó historia clínica estandarizada, incluyendo historia familiar de ECV, cuestionario para la clasificación de consumidores de cigarrillo $(\mathrm{C} 4)^{13}$, cuestionario de identificación de los trastornos debidos al consumo de alcohol (AUDIT) ${ }^{14}$ y cuestionario internacional de actividad física (IPAC) ${ }^{15}$, y se determinó la ingesta diaria de $\mathrm{Mg}$ considerando el promedio de la ingesta de alimentos de la recopilación de tres 
recordatorios de 24 horas (uno en fin de semana y dos en días laborables) ${ }^{16}$. De acuerdo con los National Institutes of Health $(\mathrm{NIH})$, se tomó como ingesta diaria recomendada (IDR) 310-320 mg/día en mujeres y $400-420 \mathrm{mg} /$ día en hombres ${ }^{17}$.

Las medidas antropométricas (peso y talla) fueron evaluadas de manera estandarizada, en condiciones de ayuno, con ropa ligera y sin zapatos, se determinaron utilizando una balanza digital electrónica Omron hbf-514 con una capacidad de $200 \mathrm{~kg} \pm 100 \mathrm{~g}$ y estadímetro marca Ade. Se calculó el IMC mediante la fórmula de Quetelet (peso en kilogramos dividido por la altura en metros al cuadrado). La TAS y TAD se determinaron con un kit de baumanómetro aneroide Hergom en los sujetos en posición sentada y después de 5 minutos de reposo, de acuerdo con la Norma Oficial Mexicana (NOM-030-1999-SSA2) para prevención, tratamiento, y control de la hipertensión ${ }^{18}$.

Se determinó: glucosa, TG, CT, HDL, LDL, PCR-us y $\mathrm{Mg}$ sérico mediante métodos estandarizados del laboratorio del Hospital Universitario de Puebla. La VLDL se estimó dividiendo TG entre 5. Fue considerada hipomagnesemia si los niveles de $\mathrm{Mg}$ sérico eran $<1.7 \mathrm{mg} / \mathrm{dl}$, normomagnesemia entre $1.7 \mathrm{y}$ $2.2 \mathrm{mg} / \mathrm{dl}$ e hipermagnesemia $>2.2 \mathrm{mg} / \mathrm{dl}^{19}$. El diagnóstico de diabetes se realizó de acuerdo con las recomendaciones de la Asociación Americana de Diabetes $^{20}$.

Se definieron como factores de mayor influencia en el RCV a aquellos factores clínico-antropométricos y bioquímicos cuantitativos que resultaron con valores de factor más altos en la componente uno y en la componente dos del ACP sin rotación varimax, utilizando TAS, TAD, IMC, glucosa TG, CT, LDL, HDL, VLDL y PCR-us.

Se describe a la población de acuerdo con el sexo. Se probó la normalidad de los datos mediante la prueba de Kolmogorov-Smirnov. Se realizó correlación de Spearman entre Mg sérico, ingesta de Mg y los factores más influyentes de RCV determinados mediante el ACP ajustado para IMC, glucosa y edad. Finalmente, debido a que el colesterol fue el factor de mayor influencia en el RCV, se comparó su concentración, así como la de $\mathrm{Mg}$ sérico y la ingesta diaria de Mg en la población dividida por sexos, presencia de tabaquismo no dependientes de cigarrillo y la ausencia de este, antecedentes familiares de ECV, obesidad y/o sobrepeso por IMC ${ }^{21}$, con y sin diabetes ${ }^{22}$; la comparación se realizó mediante t de Student para CT y U de Mann-Whitney para Mg sérico e ingesta de Mg. Además se comparó la actividad física dividida en baja, moderada y alta $^{15}$, la diferencia de concentraciones se determinó mediante ANOVA. Fue considerada una actividad física baja cuando existieron valores de MET-minutos/semana menores a 600; actividad física moderada con un total mínimo de al menos 600 METminutos/semana y actividad física alta a partir de 1,500 MET-minutos/semana ${ }^{15}$.

Debido a que el tamaño de la muestra fue a conveniencia, a posteriori se calculó el error de tipo 2 (b) de la correlación entre $\mathrm{Mg}$ y las variables que mostraron mayor relevancia (TG, VLDL, LDL y CT) utilizando el tamaño del efecto $\mathrm{w} / \mathrm{f}$ mediante $\mathrm{G}^{*}$ Power versión 3.1.9.7, resultando una potencia de $1-\beta$ de $0.97,0.97,0.99$ y 0.96 , y un error de tipo $2(\beta)$ de 0.03 , $0.03,0.01$ y 0.04 , respectivamente. El análisis estadístico se llevó a cabo en SPSS (Statistical Package for de Social Sciences) versión 23. Se consideró el valor de $\mathrm{p}<0.05$ como un resultado estadísticamente significativo.

\section{RESULTADOS}

La población de estudio estuvo conformada por 66 individuos, 53\% mujeres y $47 \%$ hombres; el $95.5 \%$ de la población fue normomagnesémica, con una mediana de $\mathrm{Mg}$ sérico de $2.2(2.1-2.3) \mathrm{mg} / \mathrm{dl}$ y una ingesta diaria de $\mathrm{Mg}$ de 282.70 (214.59-367.96) mg/ día, siendo el $69.7 \%$ los que no alcanzaron la IDR de Mg/día y el $42.4 \%$ presentó hipercolesterolemia. En la tabla 1 se muestra la caracterización clínico-antropométrica y bioquímica de la población.

El resultado del análisis de los $F R C$ cuantitativos (TAS, TAD, TG, glucosa, IMC, CT, HDL, LDL, VLDL y PCR-us) mediante ACP se observa en la tabla 2, identificándose cuatro componentes que explicaron una varianza acumulada del $76.26 \%$. La primera componente explica el $25.58 \%$ de la varianza total de los datos, resultando una influencia importante 
Tabla 1. Caracterización clínica, antropométrica y bioquímica de la población

\begin{tabular}{|c|c|c|c|}
\hline & $\mathrm{n}=66$ & $\begin{array}{c}\text { Mujeres } \\
\mathrm{n}=35\end{array}$ & $\begin{array}{c}\text { Hombres } \\
\mathrm{n}=31\end{array}$ \\
\hline Edad (años) & $44.50(35.75-56.25)$ & $41(35-56)$ & $49(40-57)^{*}$ \\
\hline $\mathrm{IMC}\left(\mathrm{kg} / \mathrm{m}^{2}\right)$ & $27.56 \pm 3.73$ & $28.08 \pm 3.93$ & $26.97 \pm 3.4^{\dagger}$ \\
\hline TAS (mmHg) & $120(110-125)$ & $110(105-120)$ & $120(110-125)^{*}$ \\
\hline $\mathrm{TAD}(\mathrm{mmHg})$ & $80(70-80)$ & $75(70-80)$ & $80(70-85)^{*}$ \\
\hline Glucosa en ayuno (mg/dl) & $94(89-104)$ & $92(87-100)$ & $98(91-109)^{*}$ \\
\hline $\mathrm{TG}(\mathrm{mg} / \mathrm{dl})$ & $149.5(109.75-220)$ & $141(109-195)$ & $150(110-259)^{*}$ \\
\hline $\mathrm{CT}(\mathrm{mg} / \mathrm{dl})$ & $193.70 \pm 39.12$ & $194.63 \pm 33.03$ & $192.65 \pm 45.57^{\dagger}$ \\
\hline $\mathrm{HDL}(\mathrm{mg} / \mathrm{dl})$ & $44.60(40.38-52.88)$ & $48(42-55)$ & $42(38-49)^{*}$ \\
\hline $\mathrm{LDL}(\mathrm{mg} / \mathrm{dl})$ & $111.37 \pm 31.19$ & $112.83 \pm 27.34$ & $109.72 \pm 35.43^{+}$ \\
\hline $\mathrm{VLDL}(\mathrm{mg} / \mathrm{dl})$ & $29.90(21.95-44)$ & $28(22-39)$ & $30(22-52)^{*}$ \\
\hline PCR-us (mg/dl) & $0.13(0.07-0.32)$ & $0.21(0.08-0.36)$ & $0.09(0.05-0.19)^{*}$ \\
\hline
\end{tabular}

*Los datos se presentan como media \pm DS para las variables con distribución normal.

†Los datos se presentan como mediana (cuartil 25\% - cuartil 75\%) para las variables sin distribución normal.

DS: desviación estándar; IMC: índice de masa corporal; TAS: tensión arterial sistólica; TAD: tensión arterial diastólica; TG: triglicéridos; CT: colesterol total; HDL: lipoproteína de alta densidad; LDL: lipoproteína de baja densidad; VLDL: lipoproteína de muy baja densidad; PCR-us: proteína C reactiva ultrasensible.

Tabla 2. Análisis de componentes principales* de las variables bioquímicas y antropométricas

\begin{tabular}{lcccc}
\hline Variables & \multicolumn{3}{c}{ Componente } \\
\cline { 2 - 5 } & 1 & 2 & 3 & 4 \\
\hline \% de varianza & 25.586 & 21.466 & 15.549 & 13.661 \\
\hline \% de varianza acumulado & 25.586 & 47.052 & 62.601 & 76.262 \\
\hline IMC & 0.087 & -0.379 & 0.316 & 0.661 \\
\hline TAS & 0.281 & -0.552 & 0.566 & -0.316 \\
\hline TAD & 0.346 & -0.386 & 0.655 & 0.395 \\
\hline Glucosa & 0.322 & -0.303 & -0.184 & -0.040 \\
\hline CT & 0.933 & -0.059 & -0.235 & 0.103 \\
\hline HDL & 0.606 & 0.740 & 0.261 & -0.208 \\
\hline LDL & -0.253 & 0.531 & 0.463 & 0.230 \\
\hline VLDL & 0.261 & 0.770 & -0.305 & -0.040 \\
\hline PCR-us & 0.933 & -0.059 & 0.431 & 0.647 \\
\hline
\end{tabular}

*Método de extracción: análisis de componentes principales. Se presenta \% de varianza por componentes y acumulado.

TAS: tensión arterial sistólica; TAD: tensión arterial diastólica; TG: triglicéridos; CT: colesterol total; HDL: colesterol de alta densidad; LDL: colesterol de baja densidad; VLDL: colesterol de muy baja densidad; PCR-us: proteína C reactiva ultrasensible.

en el RCV la que ejercen los TG (0.933) y la VLDL (0.933). La segunda componente explica el $21.46 \%$, siendo las variables más influyentes LDL $(0.770)$ y CT (0.740). Derivado de este análisis, se estableció, de acuerdo con la definición operativa, que las variables de mayor influencia en el RCV en nuestra población de estudio eran TG, VLDL, LDL y CT.

Posteriormente se identificó que el $\mathrm{Mg}$ sérico se correlaciona de manera positiva con las variables de mayor influencia para el RCV (Tabla 3). Una vez realizado el ajuste con IMC, glucosa y edad, la correlación entre Mg sérico y $\mathrm{CT}$ se mantiene positiva y significativa (Tabla 4). A posteriori se probó el poder estadístico de la correlación entre Mg y las variables que resultaron más importantes en los análisis, mismo que fue satisfactorio para todos los resultados con una potencia de $1-\beta$ de $0.97,0.97,0.99$ y 0.96 para TG, VLDL, LDL y CT, respectivamente. 
Tabla 3. Correlación entre las variables

\begin{tabular}{|c|c|c|c|c|c|c|}
\hline & Mg sérico & Ingesta $\mathrm{Mg}$ & TG & $\mathrm{CT}$ & LDL & VLDL \\
\hline $\begin{array}{l}\text { Mg sérico } \\
\text { Rho }\end{array}$ & 1.000 & 0.044 & $0.270^{*}$ & $0.349^{\dagger}$ & $0.251^{*}$ & $0.270^{*}$ \\
\hline$p$ & & 0.726 & 0.028 & 0.004 & 0.042 & 0.028 \\
\hline $\begin{array}{l}\text { Ingesta Mg } \\
\text { Rho }\end{array}$ & & 1.000 & 0.099 & 0.018 & -0.056 & 0.099 \\
\hline$p$ & & & 0.429 & 0.884 & 0.656 & 0.429 \\
\hline $\begin{array}{l}\text { TG } \\
\text { Rho }\end{array}$ & & & 1.000 & $0.444^{\dagger}$ & 0.108 & 1.000 \\
\hline $\mathrm{p}$ & & & & 0.000 & 0.389 & \\
\hline $\begin{array}{l}\mathrm{CT} \\
\text { Rho }\end{array}$ & & & & 1.000 & $0.853^{\dagger}$ & $0.444^{+}$ \\
\hline $\mathrm{p}$ & & & & & 0.000 & 0.000 \\
\hline $\begin{array}{l}\text { LDL } \\
\text { Rho }\end{array}$ & & & & & 1.000 & 0.108 \\
\hline $\mathrm{p}$ & & & & & & 0.389 \\
\hline $\begin{array}{l}\text { VLDL } \\
\text { Rho }\end{array}$ & & & & & & 1.000 \\
\hline
\end{tabular}

*Correlación de Spearman (Rho). Significación estadística $p<0.05$.

${ }^{\dagger}$ Correlación de Spearman (Rho). Significación estadística $p<0.01$.

Mg: magnesio; TG: triglicéridos; CT: colesterol total; LDL: colesterol de baja densidad; VLDL: colesterol de muy baja densidad.

Tabla 4. Correlación de magnesio con las variables influyentes de riesgo cardiovascular ajustado a índice de masa corporal, glucosa y edad*

\begin{tabular}{|c|c|c|c|c|c|c|}
\hline & Mg sérico & Ingesta $\mathrm{Mg}$ & TG & CT & LDL & VLDL \\
\hline Mg sérico & 1.000 & 0.048 & 0.246 & 0.303 & 0.234 & 0.246 \\
\hline$p$ & & 0.711 & 0.052 & 0.016 & 0.065 & 0.052 \\
\hline Ingesta Mg & & 1.000 & 0.203 & 0.015 & -0.040 & 0.203 \\
\hline $\mathrm{p}$ & & & 0.111 & 0.907 & 0.755 & 0.111 \\
\hline $\mathrm{TG}$ & & & 1.000 & 0.499 & 0.078 & 1.000 \\
\hline$p$ & & & & 0.000 & 0.541 & \\
\hline $\mathrm{CT}$ & & & & 1.000 & 0.863 & 0.499 \\
\hline$p$ & & & & & 0.000 & 0.000 \\
\hline $\mathrm{LDL}$ & & & & & 1.000 & 0.078 \\
\hline$p$ & & & & & & 0.541 \\
\hline VLDL & & & & & & 1.000 \\
\hline
\end{tabular}

*Coeficiente de correlación parcial ajustado a $\left(\mathrm{r}_{123}\right)$. Significación estadística $\mathrm{p}<0.05$.

Mg: magnesio; TG: triglicéridos; CT: colesterol total; LDL: lipoproteína de baja densidad; VLDL: lipoproteína de muy baja densidad.

Con respecto a otros estados de riesgo clínico-antropométricos que han sido asociados con mayor RCV, no se encontró diferencia significativa $(p=0.681)$ en $\mathrm{Mg}$ sérico entre mujeres (2.2 [2.1-2.3] $\mathrm{mg} / \mathrm{dl})$ y hombres $(2.2[2.1-2.3] \mathrm{mg} / \mathrm{dl}]$, tampoco se halló diferencia significativa $(p=0.839)$ en el CT entre mujeres $(194.63 \pm 33.03 \mathrm{mg} / \mathrm{dl})$ y hombres $(192.65 \pm 45.57 \mathrm{mg} / \mathrm{dl})$, pero sí se encontró diferencia significativa $(\mathrm{p}=0.005)$ en la ingesta diaria de Mg por sexos, siendo de 247.16 (190.0-337.66) mg/día en mujeres y de 338.0 (253.69-500.03) mg/día en hombres.

Se encontraron 17 fumadores no dependientes de cigarrillo, de los cuales, 7 fueron mujeres (17.1\%) y 11 hombres (35.5\%), en este caso no se hallaron 
diferencias significativas ( $p=0.791$ ) en el Mg sérico entre fumadores no dependientes de cigarrillo (2.2 [2.1-2.3] $\mathrm{mg} / \mathrm{dl})$ y no fumadores $(2.2$ [2.1-2.3] mg/dl]), así como tampoco existió diferencia significativa ( $p=0.797)$ en la ingesta de Mg entre los grupos, 278.99 (216.07-363.71) mg/día en no fumadores y 338.70 (201.74-413.21) mg/día en fumadores no dependientes de cigarrillo, y tampoco existió diferencia significativa en el CT $(p=0.123)$ entre ellos, detectándose este con $198.08 \pm 39.14 \mathrm{mg} / \mathrm{dl}$ en no fumadores y $181.06 \pm 37.31 \mathrm{mg} / \mathrm{dl}$ en fumadores no dependientes de cigarrillo.

Por otra parte, el $12 \%$ de los sujetos tenía antecedentes familiares con ECV, detectándose que la concentración de Mg sérico era de 2.15 (2.1-2.27) mg/dl, y el resto de los sujetos sin antecedentes familiares de ECV presentaron $2.2(2.1-2.2) \mathrm{mg} / \mathrm{dl}$, sin hallar diferencia significativa entre ellos $(p=0.331)$. Con respecto a la ingesta de $\mathrm{Mg}$, el grupo con antecedentes familiares de ECV consumía 222.20 (156.39271.03) mg/día, a diferencia del grupo sin antecedentes, quienes presentaban una ingesta mayor de 295.85 (220.70-385.66) mg/día, hallándose diferencia significativa entre ellos $(p=0.023)$. Y con respecto al CT, se detectó con $195.16 \pm 38.12 \mathrm{mg} / \mathrm{dl}$ en el grupo con antecedentes familiares de ECV y $183.13 \pm 47.24 \mathrm{mg} / \mathrm{dl}$ sin historia familiar, y no existió diferencia significativa entre ellos $(p=0.419)$.

Se detectó que el $72.7 \%$ de los sujetos de la población presentó obesidad y/o sobrepeso, el grupo con sobrepeso con una concentración de $2.2(2.1-2.3) \mathrm{mg} / \mathrm{dl}$ de $\mathrm{Mg}$ sérico, una ingesta de $\mathrm{Mg}$ de 282.30 (223.09389.42) mg/día y CT de $195.50 \pm 39.60 \mathrm{mg} / \mathrm{dl}$; en cambio, el grupo que padecía obesidad presentó 2.2 (2.12-2.30) $\mathrm{mg} / \mathrm{dl}$ de $\mathrm{Mg}$ sérico, su ingesta de $\mathrm{Mg}$ fue de 279.10 (214.39-350.71) $\mathrm{mg} /$ día y CT de $194.43 \pm 38.76 \mathrm{mg} / \mathrm{dl}$, sin encontrarse diferencias significativas entre ambos grupos en el $\mathrm{Mg}$ sérico, la ingesta y el $C T$ ( $p=0.589, p=0.983$ y $p=0.936$, respectivamente).

Se detectaron 14 sujetos con diagnóstico reciente de diabetes (21.2\%) y sujetos sin diagnóstico de diabetes $(n=52)$. Se halló en los portadores de diabetes una concentración de $\mathrm{Mg}$ sérico de 2.15 (1.97-2.32) $\mathrm{mg} / \mathrm{dl}$ y una ingesta de 287.30 (220.50362.67), en cambio los sujetos sin diagnóstico de diabetes presentaron $2.2(2.1-2.3) \mathrm{mg} / \mathrm{dl}$ de $\mathrm{Mg}$ sérico y una ingesta diaria de $\mathrm{Mg}$ de 282.70 (209.97374.65) mg/día, sin encontrarse diferencia significativa entre ambos grupos en ninguna de las dos mediciones ( $p=0.132$ y $p=0.937$, respectivamente); así mismo, no se hallaron diferencias significativas $(p=0.780)$ entre el CT en sujetos sin diagnóstico de diabetes $(194.40 \pm 39.72 \mathrm{mg} / \mathrm{dl})$ y los sujetos portadores de diabetes $(191.07 \pm 38.09 \mathrm{mg} / \mathrm{dl})$.

Finalmente, con respecto a la actividad física se detectó concentración de Mg sérico de $2.20 \pm 0.13 \mathrm{mg} / \mathrm{dl}$ en sujetos con actividad física moderada; $2.17 \pm 0.15 \mathrm{mg} / \mathrm{dl}$, en sujetos con actividad física alta y $2.25 \pm 0.13 \mathrm{mg} / \mathrm{dl}$ en sujetos con actividad física baja, respectivamente, sin hallarse diferencia significativa entre ellos ( $p=0.402)$; al igual que en la ingesta diaria de $\mathrm{Mg}$, siendo para sujetos con actividad física baja de $311.96 \pm 132.82 \mathrm{mg} /$ día, para aquellos con actividad física moderada de $273.83 \pm 99.17 \mathrm{mg} /$ día y los que tuvieron actividad física alta tuvieron una ingesta diaria de Mg de 385.39 mg/día, sin encontrarse diferencia significativa entre los grupos $(p=0.118)$.

En cuanto a la concentración de colesterol detectado entre los grupos de sujetos con actividad física baja, moderada y alta (191.03 $\pm 36.34 \mathrm{mg} / \mathrm{dl}$, $199.48 \pm 39.56 \mathrm{mg} / \mathrm{dl}$ y $193.80 \pm 39.12 \mathrm{mg} / \mathrm{dl} \mathrm{mg} / \mathrm{dl}$ ), tampoco se encontraron diferencias significativas $(p=0.721)$.

\section{DISCUSIÓN}

Mediante $\mathrm{ACP}$, en una población con un $95 \%$ de sujetos con normomagnesemia y conformada por hombres y mujeres de 23 a 70 años de edad, se demostró que los factores más influyentes para el RCV son los TG seguidos del CT, los cuales correlacionaron positiva y significativamente con los niveles séricos de $\mathrm{Mg}$, permaneciendo la correlación significativa y positiva con CT incluso después de ajustar a IMC, niveles de glucosa y edad ${ }^{23-25}$.

El presente estudio se centró en determinar los factores más influyentes y significativos de RCV a través del ACP para reducir 10 variables interrelacionadas en grupos de componentes independientes, 
resultando cuatro componentes. La componente uno, que representa la mayor parte de las variaciones, está fuertemente cargada de factores relacionados con hipertrigliceridemia (niveles de TG y VLDL); mientras que en la segunda componente los factores más destacados reflejaron rasgos de fenotipos de hipercolesterolemia a expensas de LDL. Por lo tanto, en el presente estudio se demostró por medio del ACP que los niveles de TG y de CT representan mayor variabilidad, incluso, que la presión arterial y la obesidad (componentes 3 y 4, respectivamente), por lo que podemos considerarlos como los factores más influyentes para el RCV en nuestra población. Por lo tanto, nuestros hallazgos confirman reportes previos en los cuales se ha encontrado asociación de riesgo entre el CT y altas tasas de incidencia de ECV relacionadas con cambios en la capa íntima y media de las arterias, así como presencia de lesiones ateroescleróticas ${ }^{5}$, por lo que los lípidos se consideran los FRC más comunes en población mexicana, confirmado también en estudios que reportan el hallazgo de una muy alta prevalencia de varias formas de dislipidemia en adultos mexicanos, especialmente en estados de hipertrigliceridemia ${ }^{26,27}$, esto mismo reportado también en otras poblaciones ${ }^{28,29}$. Es sabido que los TG representan un riesgo mayor para ECV y que estos se asocian con cifras elevadas de $\mathrm{LDL}$, aumentando el riesgo en un periodo de cinco años ${ }^{30}$; aunado a esto, la VLDL se ha identificado como un factor de riesgo para ECV aterosclerótica ${ }^{31}$.

En el presente estudio, además se estableció que los niveles séricos de Mg correlacionan positiva y significativamente con TG, VLDL, CT y LDL, sin correlacionar con HDL; y se demostró que después de ajustar por edad, IMC y glucosa de ayuno, los niveles de Mg sérico solamente correlacionaron positiva y significativamente con el CT, datos que se ven fortalecidos por la potencia obtenida en las pruebas estadísticas. Nuestros resultados son similares a los de Randell, et al. ${ }^{24}$, quienes reportaron correlación positiva de niveles séricos de $\mathrm{Mg}$ con CT y con LDL en sujetos canadienses aparentemente sanos de 19 a 62 años, proponiendo además que los niveles de Mg y lípidos difiere entre sujetos sanos y pacientes con diabetes. En nuestra población no se encontró esta diferencia, lo cual pudiera deberse a que los sujetos con diabetes fueron diagnosticados durante el estudio y las concentraciones séricas de $\mathrm{Mg}$ se encontraron dentro de los valores normales.

Contrario a nuestros resultados en población del centro de México, en población adulta del norte de México se ha reportado correlación positiva entre niveles de Mg sérico y niveles de HDL en pacientes con diabetes o intolerancia oral a la glucosa, pero no en sujetos con glucemia normal ${ }^{32}$; y se ha demostrado correlación positiva de Mg sérico con $\mathrm{HDL}$ y correlación negativa con niveles de TG y CT entre sujetos con y sin síndrome metabólico ${ }^{33}$.

Estos resultados contradictorios podrían deberse a diferencias en el metabolismo de los lípidos relacionadas con las etnias, lo cual se ha reportado en pacientes portadores de diabetes de Sudáfrica en quienes se determinó relación lineal positiva y significativa entre niveles de $\mathrm{Mg}$ y $\mathrm{CT}$, así como entre Mg y LDL, pero no entre Mg y HDL y TG, aparentemente como consecuencia de menor grasa visceral que en mujeres caucásicas ${ }^{34}$. Aunado a esto, en población china después de ajustar a factores confusores, niveles elevados de $\mathrm{Mg}$ sérico fueron asociados con niveles elevados de CT, HDL, LDL y TG, como consecuencia de la afinidad del Mg por ciertos fosfolípidos ${ }^{35,36}$. La albúmina sérica se ha relacionado también de manera lineal y directa con el Mg sérico ${ }^{37}$, por lo que consideramos relevante incluirla en estudios posteriores.

Un hallazgo sorprendente en esta población de estudio es que el $95 \%$ se encontraba normomagnesémico y el $4.5 \%$ hipermagnesémico, a pesar de que el $69.7 \%$ de la población no alcanzó la IDR de $\mathrm{Mg}^{17}$; de manera similar a nuestros resultados, en Europa y América del norte la ingesta de Mg es generalmente menor a la IDR ${ }^{38,39}$, por lo que podemos suponer que en nuestra población de estudio la absorción intestinal de $\mathrm{Mg}$ no se encuentra alterada (esto basado en que, del total de $\mathrm{Mg}$ consumido, solo del 35 al $40 \%$ es absorbido en el intestino y el resto es eliminado por las heces, y que solo el $0.1 \%$ del total del Mg se encuentra en suero $)^{40}$.

Fundamentados en lo anterior y en que no se encontró correlación significativa entre la ingesta diaria con el $\mathrm{Mg}$ en suero en este estudio, probablemente el aumento en el consumo de $\mathrm{Mg}$ no tenga beneficios sobre el RCV en población mexicana 
normomagnesémica. Este razonamiento también está basado en los resultados aportados por Song, et al. ${ }^{41}$, quienes después del ajuste por edad, IMC, ingesta de energía total, estatus de fumador, ejercicio, consumo de alcohol, uso de hormonas posmenopáusicas, multivitamínicos, diabetes, hipertensión, hipercolesterolemia e historia familiar de infarto al miocardio, y categorizadas de acuerdo con su consumo de Mg/día, reportaron que una mayor ingesta de $\mathrm{Mg}$ se asoció con un riesgo ligeramente menor de ECV, pero el RR y la tendencia lineal no fueron significativos. Estos hallazgos son contrarios a lo reportado por otros estudios, en los que el aumento en el consumo de Mg se asocia con menor RCV; sin embargo, en la mayoría de estos estudios el aumento en la ingesta se relacionó solamente con riesgo de mortalidad y no con todos los eventos cardiovasculares $^{42,43}$.

Por otra parte, la IDR de Mg es distinta para cada sexo ${ }^{17}$, sin embargo, se detectó que la IDR no fue alcanzada en mujeres ni en hombres. En cuanto a la inexistencia de diferencias de concentración de $\mathrm{Mg}$ sérico, ingesta de $\mathrm{Mg}$ y $\mathrm{CT}$ entre grupos con presencia y ausencia de tabaquismo, podría deberse a que los fumadores de nuestra población fueron no dependientes de cigarrillo, pues se sabe que la concentración de $\mathrm{Mg}$ sérico disminuye en sujetos que fuman, siendo afectado por el número de cigarrillos y la duración del hábito tabáquico ${ }^{44}$, por lo que consideramos necesario realizar estudios con fumadores dependientes para confirmar nuestros resultados. El hallazgo de una ingesta diaria de Mg significativamente menor en sujetos con antecedentes familiares de RCV que aquellos sin antecedentes permite pronosticar que una baja ingesta de Mg podría posteriormente manifestarse como una hipomagnesemia y convertirse en un factor que se sume a los factores de mayor influencia y contribuir aún más al desarrollo de ECV, pues los antecedentes familiares actúan como un medio de propensión a padecer enfermedades por la carga e interacción de rasgos genéticos y ambientales compartidos en el ambiente familiar y que son muy difíciles de eliminar ${ }^{45}$.

Con respecto a los grupos con obesidad y/o sobrepeso, no existieron diferencias significativas, sin embargo, inferimos que esto se debe a los niveles de $\mathrm{Mg}$ sérico normales en ambos grupos, sin desestimar que si existiera una mayor ingesta de Mg en ellos, podrían disminuirse marcadores importantes de adiposidad, pues se ha demostrado que con una mayor ingesta de $\mathrm{Mg}$ puede disminuir el $\mathrm{IMC}^{23}$. Con respecto a los pacientes portadores de diabetes y aquellos sin diagnóstico de diabetes, podría asumirse la ausencia de diferencias en nuestro estudio, dado que los sujetos que resultaron con diabetes tipo 2 fueron detectados como de reciente diagnóstico, además nuestra población fue mayormente normomagnesémica y la hipomagnesemia es comúnmente detectada en estados de diabetes tardía como consecuencia de la hiperglucemia ${ }^{32}$.

Finalmente, la inexistencia de diferencias entre grupos divididos por actividad física baja, moderada y alta, coinciden con un estudio realizado por Muñoz, et al. ${ }^{46}$, con sujetos atletas y sedentarios, en el que se encontraron niveles de $\mathrm{Mg}$ dentro de los valores normales, tal como en nuestra población, manifestándose incluso una menor eliminación de Mg por la orina en los atletas, por el contrario, otros estudios han declarado una disminución de $\mathrm{Mg}$ sérico después del ejercicio ${ }^{47,48}$; sin embargo, el porcentaje de sujetos en nuestro estudio con actividad física alta fue muy bajo, por lo que habría que realizar estudios incluyendo a atletas para hallar si existen diferencias en sus niveles de Mg sérico con respecto a sujetos sedentarios y observar su comportamiento ante los otros FRC con mayor influencia encontrados en esta población.

Con nuestros resultados, se puede concluir que en una población mayormente normomagnesémica, con consumo menor al requerido, el Mg sérico se correlaciona significativa y positivamente con los niveles de CT una vez ajustada a edad, IMC y niveles de glucosa, lo cual, de acuerdo con otros reportes, podría ser consecuencia de factores étnicos o de la afinidad del Mg por los fosfolípidos. Sin embargo, es necesario realizar estudios enzimáticos y de transporte de Mg para comprender la correlación encontrada e identificar si un aumento en la ingesta de Mg contribuye a una disminución de CT.

Algunas limitaciones de nuestro estudio merecen consideración. Primero, el diseño transversal del estudio dificultó determinar la dirección de la 
causalidad. Segundo, los participantes en nuestro estudio fueron reclutados voluntariamente de la comunidad, en lugar de realizar una selección al azar; sin embargo, la potencia alcanzada en las pruebas de esta población fue elevada, lo que fortalece los datos obtenidos, dado que disminuye el error de tipo 2 ( $\beta$ ) en $0.03,0.03,0.01$ y 0.04 para TG, $\mathrm{VLDL}, \mathrm{LDL}$ y $\mathrm{CT}$, respectivamente. Finalmente, nuestra población fue mayormente normomagnesémica y con ingesta de $\mathrm{Mg}$ menor a la IDR, por lo que se recomendaría realizar estudios incluyendo sujetos con hipomagnesemia e hipermagnesemia, así como con una ingesta adecuada de Mg/día.

\section{AGRADECIMIENTOS}

Agradecemos el compromiso y la dedicación de los alumnos de la clínica "Dale un minuto a tu vida», al director del Hospital Universitario de Puebla, Dr. EuIalio Morales Palacios, así como a los químicos del laboratorio de dicho hospital, especialmente a M.C. Susana Pérez Fernández, a la administradora de Farmacias Universitarias Fleming, Mtra. María de los Ángeles Benítez Simontes, y al jefe de comercialización Farmacias Universitarias Fleming, M.B.A. Juan Miguel Roldán Flores.

Al Consejo Nacional de Ciencia y Tecnología (CONACYT) por el apoyo brindado a la M.C. Karla Hilsen García Aragón con la beca de número de registro 601491.

\section{FINANCIAMIENTO}

La presente investigación no ha recibido ninguna beca específica de agencias de los sectores públicos, comercial o con ánimo de lucro.

\section{CONFLICTO DE INTERESES}

Los autores declaran no tener conflicto de intereses.

\section{RESPONSABILIDADES ÉTICAS}

Protección de personas y animales. Los autores declaran que los procedimientos seguidos se conformaron a las normas éticas del comité de experimentación humana responsable y de acuerdo con la Asociación Médica Mundial y la Declaración de Helsinki.

Confidencialidad de los datos. Los autores declaran que han seguido los protocolos de su centro de trabajo sobre la publicación de datos de pacientes.

Derecho a la privacidad y consentimiento informado. Los autores han obtenido el consentimiento informado de los pacientes y/o sujetos referidos en el artículo. Este documento obra en poder del autor de correspondencia.

\section{BIBLIOGRAFÍA}

1. Organización Mundial de la Salud. Las 10 principales causas de defunción [Internet]. Organización Mundial de la Salud; 2018 [citado: 15/07/2020]. Disponible en: https://www.who.int/es/news-room/factsheets/detail/the-top-10-causes-of-death

2. Clerkin KJ, Fried JA, Raikhelkar J, Sayer G, Griffin JM, Masoumi A, et al. COVID-19 and cardiovascular disease. Circulation. 2020;141: 1648-55.

3. Instituto Nacional de Cardiología. Estadísticas [Internet]. México: Instituto Nacional de Cardiología [citado: 17/07/2020]. Disponible en https://www.cardiologia.org.mx/transparencia/transparencia_focalizada/estadisticas/

4. Universidad Nacional Autónoma de México. COVID-19 Comorbilidades de las defunciones [Internet]. Universidad Nacional Autónoma de México [citado: 08/07/2020]. Disponible en: https://ciga-unam.maps.arcgis.com/ apps/opsdashboard/index.html\#/73880e59efc14359abfb281d6aafb9f5

5. Castro-Juárez C, Cabrera-Pivaral C, Ramírez-García S, García-Sierra L, Morales-Pérez L, Heidi R-C. Factores de riesgo para enfermedad cardiovascular en adultos mexicanos. Rev Med MD. 2018;152-62.

6. Aristizábal D. La hipertensión en el siglo XXI: los grandes cambios que nos esperan. Rev Colomb Cardiol. 2007;14(4):181-4.

7. Instituto Nacional de Estadística y Geografía. Encuesta Nacional de Salud y Nutrición 2018. México: Instituto Nacional de Estadística y Geografía; 2018.

8. Zulet MA, Puchau B, Navarro C, Martí A, Martínez Hernández JA. Biomarcadores del estado inflamatorio: Nexo de unión con la obesidad y complicaciones asociadas. Nutr Hosp. 2007;22(5):511-27.

9. Pajuelo J, Bernui I, Rocca J, Torres L, Soto L. Marcadores bioquímicos de riesgo cardiovascular en una población adolescente femenina con sobrepeso y obesidad. An Fac Med. 2009;70(1):7-10.

10. Qu X, Jin F, Hao $\mathrm{Y}, \mathrm{Li} \mathrm{H}$, Tang $\mathrm{T}$, Wang $\mathrm{H}$, et al. Magnesium and the risk of cardiovascular events: A meta-analysis of prospective cohort studies. PLoS One. 2013;8(3):1-13.

11. Rosanoff A, Seelig MS. Comparison of mechanism and functional effects of magnesium and statin pharmaceuticals. J Am Coll Nutr. 2004;23(5): 501S-505S

12. lotti S, Wolf F, Mazur A, Maier JA. The COVID-19 pandemic: is there a role for magnesium? Hypotheses and perspectives. Magnes Res. 2020; 33(2):21-7.

13. Londoño-Pérez C, Rodríguez-Rodríguez I, Gantiva-Díaz CA. Questionnaire to classify the level of tobacco consumption young people. Divers Perspect Psicol. 2011;7(2):281-91. 
14. Babor FT, Higgins-Biddle JC, Saunders JB, Monteiro MG. The alcohol use disorders identification test. Guidelines for use in primary care. World Heal Organ Ment Heal Subst Depend. 2001;(2):1-40.

15. Fan M, Lyu J, He P. Chinese guidelines for data processing and analysis concerning the International Physical Activity Questionnaire. Zhonghua Liu Xing Bing Xue Za Zhi. 2014;35(8):961-4.

16. Instituto de Nutrición de Centro América y Panamá. Manual de instrumentos de evaluación dietética. Guatemala: INCAP MDE; 2006.

17. National Institutes of Health. Magnesio [Internet]. National Institutes of Health; 24/03/2020. Disponible en: https://ods.od.nih.gov/factsheets/ Magnesium-DatosEnEspanol/

18. Diario Oficial de la Federación. Resumen integrado de la NOM-030SSA2-2009 y Guía de tratamiento farmacologico y control de la HAS. Rev Mex Cardiol. 2012;23:4-38.

19. Weisinger JR, Bellorín-Font E. Magnesium and phosphorus. Lancet. 1998;352(9125):391-6.

20. American Diabetes Association. Classification and diagnosis of diabetes: Standards of Medical Care in Diabetes-2020. Diabetes Care. 2020;43: S14-31.

21. Secretaría de Salud. NORMA Oficial Mexicana NOM-008-SSA3-2010, Para el tratamiento integral del sobrepeso y la obesidad. México: Secretaría de Salud; 2010.

22. American Diabetes Association. 2. Diabetes Care, Standards of medical care in diabetes-2020. J Clin Appl Res Educ. 2020;43(Suppl 1):S14-S31.

23. Castellanos-Gutiérrez A, Sánchez-Pimienta TG, Carriquiry A, Da Costa THM, Ariza AC. Higher dietary magnesium intake is associated with lower body mass index, waist circumference and serum glucose in Mexican adults. Nutr J. 2018;17(1):1-8.

24. Randell EW, Mathews M, Gadag V, Zhang H, Sun G. Relationship between serum magnesium values, lipids and anthropometric risk factors. Atherosclerosis. 2008; 196:413-9.

25. Barbagallo $M$, Dominguez L. Magnesium and aging. Curr Pharm Des. 2010;16(7):832-9.

26. Aguilar-Salinas CA, Olaiz G, Valles V, Manuel Ríos Torres J, Gómez Pérez FJ, Rull JA, et al. High prevalence of low HDL cholesterol concentrations and mixed hyperlipidemia in a Mexican nationwide survey. J Lipid Res. 2001;42:1298-307.

27. Barquera S, Flores M, Olaiz-Fernández G, Monterrubio E, Villalpando S, González C, et al. Dyslipidemias and obesity in Mexico. Salud Publica Mex. 2007;49(Suppl. 3):S338-S347

28. Kannel W, Castelli W, Gordon T, McNamara P. Serum cholesterol, lipoproteins, and the risk of coronary heart disease. The Framingham study. Ann Intern Med. 1971;74(1):1-12

29. Assmann G, Schulte H. The Prospective Cardiovascular Münster (PROCAM) study: Prevalence of hyperlipidemia in persons with hypertension and/or diabetes mellitus and the relationship to coronary heart disease. Am Heart J. 1988;116(6):1713-24.

30. Heart Protection Study Collaborative Group. MRC/BHF Heart Protection Study of cholesterol lowering simvastatin in 5963 people with diabetes: a randomized controlled trial. Lancet. 2003;361(14):2005-16.

31. Ren J, Grundy SM, Liu J, Wang W, Wang M, Sun J, et al. Long-term coronary heart disease risk associated with very-low-density lipoprotein cholesterol in Chinese: The results of a 15-Year Chinese Multi-Provincial Cohort Study (CMCS). Atherosclerosis. 2010;211(1):327-32.

32. Guerrero-Romero F, Rodríguez-Morán M. Hypomagnesemia is linked to low serum HDL-cholesterol irrespective of serum glucose values. J Diabetes Complications. 2000;14:272-6.

33. Guerrero-Romero F, Rodríguez-Morán M. Low serum magnesium levels and metabolic syndrome. Acta Diabetol. 2002;39:209-13.

34. Jansen van Vuuren J, Pillay S, Jansen van Vuuren C. Relationship between magnesium and lipids in patients with diabetes mellitus. J Endocrinol Metab Diabetes South Africa. 2019;24(2):46-9.

35. Cao Y, Wang C, Guan K, Xu Y, Su YX, Chen YM. Association of magnesium in serum and urine with carotid intima-media thickness and serum lipids in middle-aged and elderly Chinese: a community-based cross-sectional study. Eur J Nutr. 2016;55:219-26.

36. Merchant T, Glonek T. 31P NMR of tissue phospholipids: competition for $\mathrm{Mg} \mathrm{2+,} \mathrm{Ca} \mathrm{2+,} \mathrm{Na} \mathrm{+} \mathrm{and} \mathrm{K+} \mathrm{cations.} \mathrm{Lipids.} \mathrm{1992;27(7):551-9.}$

37. Kroll MH, Elin RJ. Relationships between magnesium and protein concentrations in serum. Clin Chem. 1985;31(2):244-6.

38. Gröber U, Schmidt J, Kisters K. Magnesium in prevention and therapy. Nutrients. 2015;7(9):8199-226.

39. Shah NC, Shah GJ, Li Z, Jiang XC, Altura BT, Altura BM. Short-term magnesium deficiency downregulates telomerase, upregulates neutral sphingomyelinase and induces oxidative DNA damage in cardiovascula tissues: Relevance to atherogenesis, cardiovascular diseases and aging. Int J Clin Exp Med. 2014;7(3):497-514.

40. Shechter M. Magnesium and cardiovascular system. Magnes Res. 2010;23(2):60-72.

41. Song Y, Manson JAE, Cook NR, Albert CM, Buring JE, Liu S. Dietary magnesium intake and risk of cardiovascular disease among women. Am J Cardiol. 2005;96(8):1135-41

42. Fang X, Wang K, Han D, He X, Wei J, Zhao L, et al. Dietary magnesium intake and the risk of cardiovascular disease, type 2 diabetes, and allcause mortality: a dose-response meta-analysis of prospective cohort studies. BMC Med. 2016;14(210):1-13.

43. Chiuve SE, Sun Q, Curhan GC, Taylor EN, Spiegelman D, Willett WC, et al. Dietary and plasma magnesium and risk of coronary heart disease among women. J Am Heart Assoc. 2013;2:1-9.

44. Ali S, Mudawi A, Ahmed SM, Hassan BA, Abd A. Assessment of the levels of serum iron and magnesium in sudanese cigarette smokers. IOSR Pharmacy. 2013;3(4):26-30.

45. van Der Sande MAB, Walraven GEL, Milligan PJM, Banya WAS, Ceesay SM, Nyan OA. Antecedentes familiares: una oportunidad para intervenir precozmente y mejorar el control de la hipertensión, la obesidad y la diabetes. Boletín de la Organización Mundial de la Salud. 2001;5:321-8.

46. Muñoz D, Llerena F, Grijota FJ, Robles MC, Alves FJ, Maynar M. Influencia de la actividad física sobre la eliminación urinaria de minerales y elementos traza en sujetos que viven en la misma área geográfica. Rev Andaluza Med del Deport. 2018;11(1):7-11.

47. Lukaski HC. Magnesium, zinc, and chromium nutriture and physical activity. Am J Clin Nutr. 2000;72(2 Suppl.):585-93.

48. Laires MJ, Alves F. Changes in plasma, erythrocyte, and urinary magnesium with prolonged swimming exercise. Magnes Res. 1991;4(2):119-22. 